\title{
Escala Califórnia de Vitimização do Bullying (ECVB): Evidências de Validade e Consistência Interna
}

\author{
Ana Karla Silva Soares \\ Programa de Pós-Graduação em Psicologia da Universidade Federal da Paraíba, \\ João Pessoa, PB, Brasil \\ Valdiney V. Gouveia ${ }^{1}$ \\ Departamento de Psicologia da Universidade Federal da Paraíba, João Pessoa, PB, Brasil \\ Rildésia S. V. Gouveia \\ Departamento de Direito do Centro Universitário de João Pessoa, João Pessoa, PB, Brasil \\ Patrícia Nunes da Fonsêca \\ Departamento de Psicopedagogia da Universidade Federal da Paraíba, \\ João Pessoa, PB, Brasil \\ Carlos Eduardo Pimentel \\ Departamento de Psicologia da Universidade Federal da Paraíba, João Pessoa, PB, Brasil
}

\begin{abstract}
Resumo
Vitimização do Bullying (ECVB), reunindo evidências de sua validade de construto e consistência interna. Dois estudos foram realizados em João Pessoa (PB) com estudantes do ensino fundamental. No Estudo 1, participaram 297 jovens com idade média de 14 anos ( $54 \%$ do sexo feminino), os quais responderam a ECVB e perguntas demográficas. Uma análise de componentes principais revelou um único fator (valor próprio de 2,82), explicando $40,3 \%$ da variância total e apresentando alfa de Cronbach $(\alpha)$ de 0,72 . No Estudo 2, participaram 327 jovens com idade média de 12 anos (51\% do sexo feminino), que responderam os mesmos instrumentos. Por meio de análise fatorial confirmatória, corroborou-se esta estrutura unifatorial, $\chi^{2} / g l=2,34$, AGFI $=0,94, \mathrm{CFI}=0,96$, TLI $=0,94, \mathrm{RMSEA}=0,064$ (IC90\% $=0,036-0,093$ ). Este apresentou $\alpha=0,77$, tendo homogeneidade de 0,34 e confiabilidade composta de 0,78. Concluiu-se que a ECVB é uma medida que apresenta evidências de validade e precisão, podendo ser usada em estudos futuros para conhecer os correlatos do bullying.
\end{abstract}

Palavras-chave: Bullying, vitimização, escala, validade, precisão.

\section{California Victimization of Bullying Scale (CVBS): Evidence of Validity and Internal Consistency}

\begin{abstract}
This study aimed at adapting to the Brazilian context the California Bullying Victimization Scale (CVBS), gathering evidence of its construct validity and reliability. Two studies were conducted in

Endereço para correspondência: Universidade Federal da Paraíba, Centro de Ciências Humanas, Letras e Artes, Departamento de Psicologia, Núcleo de Pesquisa Bases Normativas do Comportamento Social, Bloco C, $2^{\text {a }}$ andar, Sala 1, João Pessoa, PB, Brasil 58051-900. E-mail: akssoares@gmail.com, vvgouveia@gmail.com, rsvgouveia@gmail.com, patynfonseca@hotmail.com e kdu1976@gmail.com

O presente estudo contou com apoio do Conselho Nacional de Desenvolvimento Científico e Tecnológico (CNPq) por meio de bolsa de Produtividade em Pesquisa ao segundo autor. A primeira conta com bolsas da Coordenação de Aperfeiçoamento de Pessoal de Nível Superior (CAPES) de doutorado.
\end{abstract}


João Pessoa (PB) with elementary school students. In Study 1 participants were 297 young people with a mean age of 14 years (54\% female), who answered CVBS and demographic questions. A principal components analysis revealed a single factor (eigenvalue of 2.82 ), accounting for $40.3 \%$ of the total variance and Cronbach's alpha $(\alpha)$ of .72 . In Study 2 participated 327 young people with a mean age of 12 years $(51 \%$ female) who answered the same instruments. A confirmatory factor analysis corroborated this one-factor structure, $\chi^{2} / d f=2.34, \mathrm{AGFI}=.94, \mathrm{CFI}=.96, \mathrm{TLI}=.94, \mathrm{RMSEA}=.064(90 \% \mathrm{CI}=.036$ to .093). This one-factor solution showed $\alpha=.77$, homogeneity of .34 and composite reliability of .78. In conclusion, the CVBS showed evidence of validity and reliability, can be used in further studies to know the correlates of bullying.

Keywords: Bullying, victimization, scale, validity, reliability.

\section{Escala California de Victimización de Bullying (ECVB): Evidencias de Validez y Consistencia Interna}

\section{Resumen}

El objetivo de este estudio ha sido adaptar al contexto brasileño la Escala California Victimización Bullying (ECVB), reuniendo evidencias de su validez de constructo y fiabilidad. Dos estudios se han realizado en João Pessoa (PB) con estudiantes de escuelas primarias. En el Estudio 1 participaron 297 jóvenes con una edad promedia de 14 años (54\% mujeres), que respondieron a la ECVB y preguntas demográficas. Un análisis de componentes principales reveló un solo factor (valor propio de 2.82), explicando un $40.3 \%$ de la varianza total, con un alfa de Cronbach $(\alpha)$ de .72. En el Estudio 2 participaron 327 jóvenes con una edad promedia de 12 años (51\% mujeres), que respondieron a los mismos instrumentos. A través de un análisis factorial confirmatorio se corroboró esta estructura unifactorial, $\chi^{2} / \mathrm{gl}=$ 2.34, $\mathrm{AGFI}=.94, \mathrm{CFI}=.96, \mathrm{TLI}=.94, \mathrm{RMSEA}=.064(\mathrm{IC} 90 \%=.036-.093)$. Este factor general mostró $\alpha=.77$, homogeneidad de .34 y fiabilidad compuesta de .78. Se concluyó que la ECVB es una medida psicometricamente adecuada, pudiendo ser utilizada en estudios futuros para conocer los correlatos del bullying.

Palabras clave: Bullying, victimización, escala, validez, fiabilidad.

Embora seja um tema que nos últimos anos tenha despertado o interesse de pesquisadores e da mídia em geral, retratado, por exemplo, por meio de filmes, novelas e programas de auditório, o estudo sistemático do bullying remete a pesquisas desenvolvidas desde já na década de 1980, principiando com os trabalhos de Dan Olweus (Universidade de Bergen). Este autor publicou o livro Aggression in the Schools: Bullies and Whipping Boys (1978), elaborando concomitantemente uma campanha antibullying em escolas norueguesas. No entanto, foi em meados dos anos 1990 que o tema ganhou notoriedade no meio acadêmico, impulsionando o desenvolvimento de pesquisas em diversas partes do mundo (Hoover, Oliver, \& Hazler, 1992; Olweus, 1996).
Incidentes envolvendo atos de violência de estudantes para com seus colegas de sala e professores, a exemplo dos ocorridos nos massacres de Columbie, em 1999, e Realengo, em 2011, ajudaram esta temática a se destacar não apenas no âmbito acadêmico, mas também na sociedade em geral. Diante destes fatos, observa-se o crescimento da necessidade de se entender a temática de maneira aprofundada e, partindo do conhecimento adquirido, elaborar uma ferramenta que possibilitasse mensurar estes comportamentos entre os jovens, por meio de uma medida que reúna parâmetros adequados de validade e precisão.

Originalmente, o conceito utilizado na Escandinávia para se referir ao bullying foi o mobbing (Olweus, 1978). No entanto, apesar de 
alguns autores tratarem os termos de forma indistinta (Zapf, 1999), segundo Leymann (1990), o mobbing é mais direcionado aos adultos no contexto ocupacional, sendo definido como uma forma de comunicação hostil e antiética, direcionada a uma pessoa como uma forma de violência psicológica. Já o termo bullying é aplicado a crianças e adolescentes, principalmente em ambiente escolar.

A palavra bullying é de origem inglesa, inexistindo na língua portuguesa uma tradução consensual a respeito, encerrando todos os atributos definidores dos comportamentos subjacentes, definido como a intenção deliberada de prejudicar a outra pessoa com a repetição do comportamento ao longo de um período de tempo e na presença de um relacionamento assimétrico entre agressor e vítima (Sairanen \& Pfeffer, 2011).

Salmivalli (2010) define o bullying como um subtipo do comportamento agressivo, em que um indivíduo ou um grupo, repetidamente, ataca, humilha e/ou exclui uma pessoa relativamente incapaz, sendo um fenômeno social que transcende gênero, idade e cultura (Sansone \& Sansone, 2008). Ele é considerado um subtipo de vitimização realizado por pares, mas se diferencia de outros tipos de vitimização pela presença de três elementos principais: intenção, repetição e desequilíbrio de poder, os quais precisam estar presentes para caracterizar este fenômeno (Felix, Sharkey, Green, Furlong, \& Tanigawa, 2011).

Segundo Vanderbilt e Augustyn (2010), o bullying geralmente está associado à afirmação de poder por meio da agressão repetida e intencional realizada pelo bully (termo em inglês para agressor) com pessoas consideradas mais fracas (vítimas). Assim, o bullying não é uma simples luta entre dois indivíduos equivalentes em força física e psicológica, mas sim uma disputa baseada no desequilíbrio de poder e na repetição destes comportamentos ao longo do tempo.

Dentre as medidas disponíveis na literatura, destaca-se o Olweus Bully/Victim Questionnaire (OBVQ), que foi desenvolvido pelo próprio Olweus (1984). Este questionário avalia o bullying e a vitimização por meio de perguntas sobre a frequência, os tipos, o local e os "valentões" que participam do bullying. Também con- templa quantas vezes os estudantes informam sobre ameaças sofridas aos familiares e professores, indicando se estes intervêm e realizam atos para inibir as intimidações.

Não obstante, apesar do OBVQ ser uma medida amplamente difundida no meio acadêmico e ter passado por revisões sucessivas desde sua elaboração (Atik, 2011; Olweus, 1996), não está isenta de críticas (Cornell, Sheras, \& Cole, 2006; Lee \& Cornell, 2010), sobretudo em razão do fato de não detalhar e publicar seus parâmetros psicométricos e por apresentar, no início das instruções, uma definição longa de bullying, dificultando sua compreensão por parte das crianças. Destaca-se, igualmente, que esta medida é extensa (40 itens), podendo produzir cansaço no participante, principalmente quando estes são infantes, que requerem medidas curtas e de fácil administração (Barbosa, Gouveia, \& Barbosa, 2003).

Uma busca recente nas bases de dados Google acadêmico (2013) e Index Psi (2013), considerando os últimos cinco anos e tendo por expressões-chave "escala de bullying" e "bullying scale", localizou inicialmente 24 ocorrências, destacando-se dentre as quais sete sobre bullying no contexto organizacional, uma no contexto prisional, uma na internet (cyberbullying), uma direcionada a avaliar o bullying contra homossexuais, uma elaborada com base nos instrumentos de Olweus (1996) e Rigby (1998), uma que empregava definição prévia de bullying, tal como a de Olweus (1996), e duas no contexto brasileiro, sendo que direcionadas a mensurar o bullying no ensino superior e outra que visava investigar a prevalência de violência escolar. Dentre estas medidas, foi identificada a Escala Califórnia de Vitimização do Bullying (ECVB; Felix et al., 2011), que apresentou evidências de validade e precisão consistentes em contexto estadunidense, mas também com tais evidências psicométricas no contexto turco (Atik \& Guneri, 2012).

Diante do anteriormente exposto, visto a necessidade de se mensurar o comportamento de bullying em crianças e adolescentes, é pertinente ter em conta a Escala Califórnia de Vitimização do Bullying (ECVB). Esta reuniu evidências de validade e precisão compatíveis com os de ou- 
tras medidas deste construto, porém não incidem nela alguns pontos críticos de outras medias (e.g., empregar definição previa de bullying, número excessivo de itens, conteúdo de compreensão difícil). Apesar destas qualidades, não se encontrou qualquer estudo acerca de sua adaptação para o contexto brasileiro, o que motivou o presente estudo. Seu objetivo principal foi adaptar a referida escala para este contexto, reunindo evidências de sua adequação psicométrica. Especificamente, procurou-se conhecer sua validade fatorial e consistência interna, descrevendo-se os estudos empíricos levados a cabo com este propósito. Previamente, entretanto, procura-se resumir a conceituação do bullying e, em seguida, apresentar os parâmetros psicométricos da ECVB.

Em suma, o bullying consiste no fenômeno por meio do qual a criança ou o adolescente é exposto de forma sistemática a um conjunto de atos agressivos, desprovidos de motivação aparente, de maneira intencional e realizados por um ou mais indivíduos (agressores) e que ocorre, com maior incidência, no ambiente escolar (Lisboa, Braga, \& Ebert, 2009; Olweus, 1996). A avaliação de sua presença tem sido feita por meio de entrevistas, porém são mais comuns os questionários e as escalas, como a que se descreve a seguir.

\section{Escala Califórnia de Vitimização do Bullying}

Felix et al. (2011) desenvolveram uma medida para avaliar o bullying em estudantes de nível fundamental e médio, composta por sete itens. Compreende um instrumento de autorrelato que avalia as diversas formas de bullying, sem empregar o termo ou definições prévias, pois estes autores consideram o termo "emocionalmente carregado", podendo influenciar as respostas das vítimas ou dos agressores. Procurando diferenciá-lo de outras formas de vitimização, elaboraram-se itens considerando os critérios de definição do bullying, ou seja, se é intencional, repetitivo em um dado período de tempo e possui o desequilíbrio de poder. Foram então elaboradas perguntas relacionadas com comportamentos correlatos como (e.g., se já foi provocado ou xingado, teve rumores ou fofocas espalhadas sobre si ou teve comentários sexuais, piadas ou gestos obscenos realizados consigo).

Com o propósito de diferençar vítimas de bullying e de pares, os participantes são convidados a indicar se consideram que estes comportamentos foram realizados de forma intencional e se os magoaram, respondendo sim ou não. Outra maneira empregada para verificar a diferença (vítima de bullying ou pares) foi considerar a presença de desequilíbrio de poder entre os envolvidos. Esta questão foi avaliada por meio de dez_adjetivos referente à percepção da vítima em termos de popularidade, inteligência e força [e.g., popular, esperto(a), bonito(a)] quando comparada ao agressor. A partir das situações apresentadas, os respondentes eram solicitados a se comparar com a pessoa que realizou tais atos com ela, respondendo em escala de 3 pontos (1 = Menos do que eu, 2 = Parecido comigo ou 3 $=$ Mais do que eu). Esta medida apresentou evidência de precisão (teste-reteste) em um período de duas semanas $\left(r=0,80\right.$ para $5^{\circ}$ e $6^{\circ}$ séries e 0,83 para $7^{\circ}$ e $8^{\circ}$ séries). A ECVB também reuniu evidências de validade convergente, tendo se correlacionado com outra medida de bullying, fazendo-o negativamente com medidas de conexão com a escola, esperança e satisfação com a vida (Felix et al., 2011).

A fim de empregar a ECVB em outro contexto, Atik e Guneri (2012) indicam previamente suas evidências de validade e precisão entre estudantes turcos. A amostra foi constituída por 313 daqueles matriculados na $6^{\circ}, 7^{\circ}$ e $8^{\circ}$ séries, os quais apresentaram idade média de 12,6 anos $(D P=0,98)$. Tais autores constataram que esta medida apresentou consistência interna aceitável (alfa de Cronbach de 0,72), assim como são identificadas evidências se sua validade convergente, avaliada por meio de sua correlação com as pontuações no questionário de bullying de Olweus ( $r=0,73, p<0,05)$, e validade discriminante, que foi aferida em razão da sua correlação com a medida de satisfação com a vida, de Siyez e Kaya, $2008(r=-0,29, p<0,05)$.

Diante do previamente exposto, constata-se que para conhecer os antecedentes e consequentes do bullying, demanda-se previamente contar 
com medida psicometricamente adequada. Neste sentido e tomando em conta os parâmetros da Escala Califórnia de Vitimização do Bullying, parece justificável o empreendimento de adaptá-la ao contexto brasileiro, reunindo evidências de validade fatorial e consistência interna que somem aos achados previamente descritos (Atik \& Guneri, 2012; Felix et al., 2011). Deste modo, procederam-se dois estudos, que são descritos a seguir.

\section{Estudo 1. Estrutura Fatorial da Escala Califórnia de Vitimização do Bullying}

Este estudo é o primeiro intento de adaptar a Escala Califórnia de Vitimização do Bullying para o contexto brasileiro, reunindo evidências de sua validade fatorial e consistência interna. Assim, procedeu-se inicialmente a uma análise fatorial a fim de explorar a estrutura fatorial na representação do construto bullying, avaliando posteriormente em que medida este fator geral apresentava consistência interna que justifique tratá-la como tal.

\section{Método}

\section{Participantes}

Participaram do presente estudo 297 estudantes de João Pessoa (PB), os quais apresentaram idade média de 14 anos $(D P=1,28$; variando de 11 a 16 anos), a maioria do sexo feminino (54\%) e de escolas públicas (56\%). Tratou-se de uma amostra de conveniência (não probabilística), contando-se com aqueles que, quando solicitados, se dispuseram a participar da pesquisa.

\section{Instrumentos}

Os participantes responderam um livreto que incluiu duas partes: a Escala Califórnia de Vitimização do Bullying (ECVB; Felix et al., 2011) e perguntas demográficas (sexo, idade, religião e religiosidade). Previamente descrita, esta medida foi originalmente elaborada em língua inglesa, sendo formada por sete itens, sendo os participantes questionados quanto à frequência com que sofrem uma série de ações conside- radas exemplos de comportamentos de bullying, devendo expressar suas respostas em escala de 5 pontos, variando de 0 (Nunca) a 4 (Várias vezes durante a semana). Em seguida a pessoa é convidada a indicar se considera estes comportamentos intencionais e importantes (se os magoaram), respondendo sim ou não. Por fim, com base nas respostas atribuídas na parte anterior, solicita-se que a pessoa se compare com a "principal pessoa que fez tais coisas", respondendo em escala de 3 pontos $(1=$ Menos do que eu, 2 = Parecido comigo e 3 = Mais do que eu).

\section{Procedimento}

Inicialmente, dois pesquisadores bilíngues realizaram a tradução do instrumento do inglês para o português, sendo em seguida empregado o método back-translation, no qual a última versão elaborada em português foi retraduzida para o inglês e, contando com o auxílio de um terceiro colaborar bilíngue, comparada com a versão original. Posteriormente, contataram-se os responsáveis pelas instituições de ensino, visando obter permissão para realização da pesquisa em suas escolas. Estes foram informados sobre os objetivos da pesquisa e, após consentimento e assinatura do termo de responsabilidade e do Termo de Consentimento Livre e Esclarecido (TCLE) por parte dos pais ou responsáveis, agendou-se o melhor horário para aplicação do questionário. A coleta de dados foi efetuada por cinco colaboradores. Embora esta tenha sido realizada em ambiente coletivo de sala de aula, a participação foi individual. Os participantes foram informados acerca do caráter voluntário da participação, garantindo-se o anonimato e o sigilo da participação, tendo o projeto sido previamente aprovado pelo Comitê de Ética em Pesquisa com Seres Humanos (Parecer n ${ }^{\circ} 88.166 / 2012$ ), seguindo-se práticas recomendadas na Resolução do Conselho Nacional de Saúde no 466/12. Em média, 10 a 15 minutos foram suficientes para concluir a participação.

No que se refere à análise dos dados, empregou-se o programa SPSS (versão 18) para analisar os dados, permitindo calcular estatísticas descritivas (medidas de tendência central e dispersão, frequência) para caracterizar os par- 
ticipantes do estudo e o teste $t$ de Student para averiguar o poder discriminativo dos itens, além da análise fatorial e consistência interna da escala, que permitiram checar as evidências de validade e precisão.

\section{Resultados}

Primeiramente, procedeu-se à análise do poder discriminativo dos itens da ECVB, partindo-se do critério de mediana empírica $(M d n=3)$ para definir os grupos-critério inferior e superior. Definidos tais grupos, calculou-se um teste $t$ de Student (amostras independentes) para cada item, comparando estas médias nos dois grupos, observando-se que todos os itens discriminaram com a magnitude e direção esperada ( $t \geq 7,84$, $p<0,001)$. Entretanto, não se observou homogeneidade nas pontuações médias, que variaram de $0,25, D P=0,65$; Item 5 . Você foi ameaçado por seu(s) colega(s)? a $0,90, D P=1,45$; Item 1. Você foi provocado ou apelidado por seu(s) $\operatorname{coleg} a(s)$ ?.
Conhecido o poder discriminativo dos itens, procurou-se identificar a estrutura fatorial subjacente à ECVB. Desta feira, efetuou-se uma análise de componentes principais, que se mostrou um procedimento justificável, $\mathrm{KMO}=0,78$ e Teste de Esfericidade de Bartlett, $\chi^{2}(21)=405,02, p$ $<0,001$; não se fez qualquer imposição quanto ao número de componentes a extrair e ao tipo de rotação. A partir de então foi possível identificar uma estrutura formada por um componente geral, cujo valor próprio (eigenvalue) foi superior a 1 (2,82; Critério de Kaiser), explicando 40,3\% da variância total. Tendo em consideração a fragilidade deste critério, procedeu-se a uma análise paralela (Hayton, Allen, \& Scarpello, 2004), admitindo-se os parâmetros do banco de dados (296 participantes e 7 itens) e efetuando 1.000 simulações. Contratando os valores próprios observados na PCA com os simulados, percebeu-se que o segundo valor próprio empírico $(0,98)$ foi inferior ao simulado $(1,12)$, confirmando a unidimensionalidade desta medida (Tabela 1).

\section{Tabela 1}

Estrutura Fatorial da Escala Califórnia de Vitimização do Bullying - ECVB

\begin{tabular}{clcc}
\hline \multicolumn{1}{c}{ Conteúdo dos itens } & Carga & $h^{2}$ \\
\hline 05 & Você foi ameaçado por seu(s) colega(s)? & 0,73 & 0,54 \\
04 & Você foi empurrado ou agredido fisicamente? & 0,72 & 0,52 \\
07 & Você teve comentários sexuais ou gestos correspondentes dirigidos a você? & 0,65 & 0,43 \\
$03 \quad$ Você foi deixado de fora do grupo ou ignorado por seu(s) colega(s)? & 0,65 & 0,43 \\
$02 \quad$ Você teve rumores, boatos ou fofocas espalhados sobre você por seu(s) & 0,62 & 0,33 \\
06 & colega(s) pelas suas costas? & 0,62 & 0,38 \\
01 & Você teve suas coisas roubadas ou danificadas por seu(s) colega(s)? & 0,58 & 0,38 \\
\hline Número de itens & & 7 & \\
Valor Próprio & 2,82 & 40,3 & 0,72 \\
\% de variância & & \\
alfa de Cronbach & &
\end{tabular}

Conforme é possível observar nesta tabela, a estrutura da medida é representada por um componente congruente com a proposta original que a fundamentou. Todos os itens apresentaram saturações superiores a 0,40 , variando de 0,58 , Item 1 . Você foi provocado ou apelidado por seu(s) colega(s)? a 0,73 , Item 5. Você foi ameaçado por seu(s) 
colega(s)?. Quanto à sua consistência interna, calcularam-se os valores de alfa de Cronbach $(\alpha=0,72)$, a homogeneidade, correlação média inter-itens, $r_{\text {i.i }}=0,30$, variando de 0,14 (Itens 3 e 7) a 0,54 (Itens 4 e 5) e a correlação Item-total, variando de 0,37 (Item 2) a 0,55 (Item 5), com média de 0,45. Diante destes resultados, optou-se por assumir uma estrutura constituída por um componente da Escala Califórnia de Vitimização do Bullying, que é coerente com a versão proposta originalmente.

\section{Discussão Parcial}

O presente estudo teve por objetivo adaptar a ECVB para o contexto brasileiro, verificando evidências de sua validade fatorial e consistência interna. Confia-se que este tenha sido cumprido. A análise do poder discriminativo dos itens possibilitou mostrar que todos funcionaram adequadamente, discriminando participantes com pontuações próximas (Pasquali, 2003). Sua estrutura fatorial, inclusive sem impor qualquer restrição, mostrou-se unidimensional, o que foi atestado pelo Critério de Horn (Hayton et al., 2004). Já seu alfa de Cronbach foi equivalente àqueles relatados em estudos prévios (Atik \& Guneri, 2012; Felix et al., 2011); constatou-se ainda que o fator (componente) geral reuniu itens que cumprem critérios de homogeneidade (Clark \& Watson, 1995). Apesar desses achados, não se deve desconsiderar que as análises empreendidas foram eminentemente exploratórias, não sendo realizada qualquer avaliação que confirmassem a estrutura unifatorial desta medida, o que motivou a realização do estudo descrito a seguir.

\section{Estudo 2. Estudo Confirmatório da Estrutura Fatorial da ECVB}

Este estudo teve por objetivo proceder a análises de natureza confirmatória, procurando complementar aquelas previamente realizadas, reduzindo possíveis dúvidas acerca dos parâmetros de validade fatorial e consistência interna desta medida. Especificamente, pretende-se efetuar uma análise fatorial confirmatória e checar evidências complementares de consistência interna, descrevendo seus indicadores de alfa de Cronbach e Confiabilidade Composta (CC).

\section{Método}

\section{Participantes}

Contou-se com a participação de 327 estudantes de instituições públicas $(80 \%)$ e privadas (20\%) de João Pessoa (PB), que apresentaram idade média de 12 anos $(D P=1,96$, variando de 9 a 16 anos). Estes foram distribuídos de forma proximamente equânime em relação ao sexo ( $51 \%$ sexo feminino), sendo que a maioria se declarou católica (58\%), obtendo uma média de religiosidade $(M=2,7, D P=1,38)$ acima do ponto médio da escala de resposta, 0 (Nada religioso) a 4 (Muito religioso). Como ocorreu no estudo anterior, tratou-se de uma amostra de conveniência (não probabilística), tendo participado aqueles que, presentes em sala de aula, concordaram em colaborar voluntariamente.

\section{Instrumentos e Procedimento}

Aos respondentes foi entregue um livreto contendo a Escala Califórnia de Vitimização do Bullying (ECVB) e perguntas demográficas (sexo, idade, religião e religiosidade), que foram descritos no Estudo 1. Foram empregados os mesmos procedimentos éticos e de coleta de dados do estudo anterior. Em média, 15 minutos foram suficientes para concluir a participação neste estudo.

\section{Análise dos Dados}

Além de empregar o SPSS (versão 18) para o cálculo de estatísticas descritivas e consistência interna, utilizou-se o AMOS (versão 18) com o fim de avaliar a estrutura fatorial desta escala. Calculou-se a confiabilidade composta (CC), cujo valor a partir de 0,70 garante evidência deste parâmetro (Fornell \& Larcker, 1981; Hair, Black, Babin, Anderson, \& Tatham, 2009). É importante esclarecer que, diferentemente do alfa de Cronbach que demanda tau equivalência, isto é, que haja saturações de tamanhos similares e erros de medidas independentes, a CC não 
faz esta suposição. No caso da análise fatorial confirmatória, tomou-se a matriz de covariância como entrada, adotando o estimador ML ( $\mathrm{Ma}$ ximum Likelihood); os seguintes indicadores de ajuste foram considerados (Tabachnick \& Fidell, 2013):

- $\chi^{2}$ (qui-quadrado). É um indicador que avalia a probabilidade de o modelo teórico se ajustar aos dados, onde valores baixos são indicadores de modelos ajustados. É importante considerá-lo para uma visão geral de modelos concorrentes, porém, devido a sua sensibilidade ao tamanho da amostra e número de variáveis do modelo, costuma-se empregar sua relação em relação aos graus de liberdade do modelo $\left(\chi^{2} / g l\right)$. No caso, este é considerado como uma qualidade subjetiva de ajuste, em que valores entre dois e três (aceitando até cinco) são indicadores de adequação do modelo teórico os dados.

- GFI (Goodness-of-Fit Index) e AGFI ( $A d$ justed Goodness-of-Fit Index). O GFI foi um indicador criado como alternativa ao $\chi^{2}$ (qui-quadrado), porém ainda apresenta sensibilidade quanto ao número elevado de graus de liberdade em relação ao tamanho da amostra. Nesta direção, o AGFI funciona como um indicador ponderado baseado nos graus de liberdade e que tende a aumentar com o tamanho da amostra. Ambos refletem a proporção de variância-covariância nos dados explicada pelo modelo; seus valores variam de 0 a 1 , aceitando-se aqueles próximos ou superiores a 0,90 como indicadores de ajuste do modelo (Hooper, Coughlan, \& Mullen, 2008).

- CFI (Comparative Fit Index). Este é considerado um índice adicional de ajuste de modelo que funciona satisfatoriamente quando o tamanho da amostra é pequeno. Constitui-se como um indicador que comparar a matriz de covariância predita pelo modelo com aquela realmente observada comparativo, com valores na casa de 0,90 expressando um ajuste adequado do modelo.

- TLI (Tucker-Lewis coeficiente). É considerado um indicador global de adequação do modelo. São considerados satisfatórios indicadores próximos de 1,00, admitindo-se valores entre 0,80 e 0,90 .

- RMSEA (Root-Mean-Square Error of Approximation), seu intervalo de confiança de $90 \%$ (IC90\%) e o Pclose. O RMSEA é considerado um indicador de "maldade de ajuste", ou seja, valores altos indicam um modelo desajustado. De acordo com Diamantopoulos e Siguaw (2000), trata-se de um dos indicadores de ajuste mais informativos e parcimoniosos, pois seleciona o modelo com menor número de parâmetros. Recomendam-se valores entre 0,05 e 0,08 , admitindo-se até 0,10 como satisfatórios, desde que caia no intervalo de $90 \%$ de confiança (IC90\%; Hooper et al., 2008). O Pclose é um indicador mais criterioso, testando a hipótese de RMSEA > 0,05, $\operatorname{com} p$ $>0,05$ sugerindo sua rejeição.

\section{Resultados}

Considerando a estrutura preconizada por Felix et al. (2011) e os achados do Estudo 1, testou-se a estrutura unifatorial da Escala Califórnia de Vitimização do Bullying com os sete itens saturando no mesmo fator geral. Os itens não seguiram distribuições perfeitamente normais uni e multivariada. No caso, os valores de skewness e kurtosis mais extremos foram observados para os itens $1(0,80$ e $-0,76$, respectivamente) e 5 ( 2,97 e 8,73 , respectivamente), e a razão crítica de normalidade multivariada foi de 60,65 . Apesar de o pressuposto de normalidade ter sido violado, procedeu-se à análise fatorial confirmatória, estimação maximuum likelihood (ML), visto que o tamanho da amostra $(n>200)$ e a razão de participantes por item (mínimo de 10) foram respeitados (Biddle, Markland, Gilbourne, Chatzisarantis, \& Sparkes, 2001). Os resultados corroboraram a dita estrutura, cujos indicadores de ajuste foram como seguem: $\chi^{2}$ (14) $=32,88, p<0,01, \chi^{2} / g l=2,34, \mathrm{GFI}=0,97$, AGFI $=0,94, \mathrm{CFI}=0,96, \mathrm{TLI}=0,94, \mathrm{RMSEA}=0,064$ (IC90\% $=0,036-0,093)$ e Pclose $=0,18$. Todos os itens apresentaram saturações (lambdas) estatisticamente diferente de zero $(\lambda \neq 0 ; z>1,96$, $p<0,001$ ), com valores variando de 0,46 , Item 
1. Você foi provocado ou apelidado por seu(s) colega(s)? a 0,73, Item 5. Você foi ameaçado por seu(s) colega(s)?. Estes resultados sugerem a adequação do modelo teórico aos dados (Figura 1).

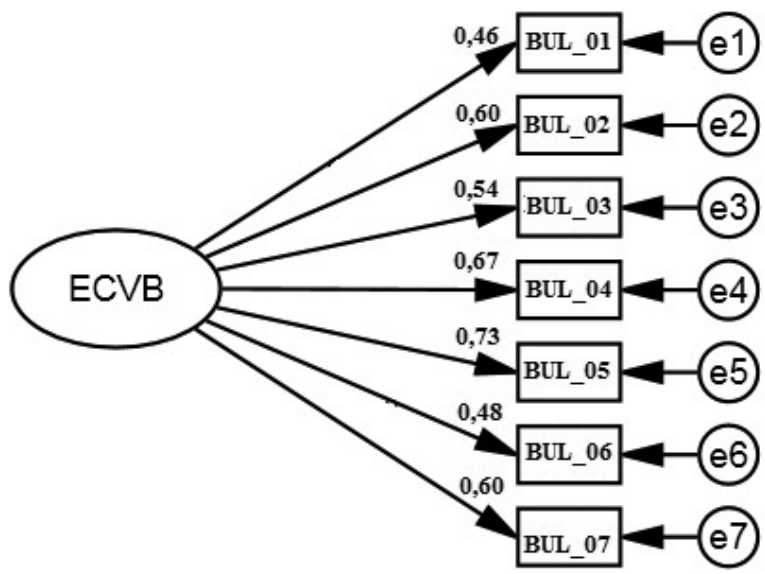

Figura 1. Estrutura Fatorial da Escala Califórnia de Vitimização do Bullying.

Quanto aos indicadores de consistência interna, calcularam-se o alfa de Cronbach $(\alpha=$ 0,77 ), a homogeneidade (correlação média inter-itens, $\left.r_{\text {i.i }}=0,34\right)$ e Confiabilidade Composta $(\mathrm{CC}=0,78)$, que, conjuntamente, corroboraram este parâmetro de consistência interna.

\section{Discussão Parcial}

Este estudo procurou reunir evidências mais robustas acerca da estrutura fatorial e consistência interna da ECVB. Os resultados corroboraram a adequação de sua estrutura unifatorial, reunindo seus sete itens, que apresentou indicadores de ajuste que cumprem os critérios preconizados na literatura (Tabachnick \& Fidell, 2013). Por fim, os múltiplos indicadores de homogeneidade foram igualmente satisfatórios, cujos valores superaram aqueles que têm sido recomendados no caso de alfa de Cronbach (Pasquali, 2003), homogeneidade (Clark \& Watson, 1995) e confiabilidade composta (Hair et al., 2009).

\section{Discussão Geral}

Os estudos realizados anteriormente tiveram como objetivo geral reunir evidências de adequação psicométrica da ECVB, avaliando sua validade (fatorial e convergente) e consistência interna (alfa de Cronbach, homogeneidade e confiabilidade composta). Deste modo, estima-se que seu propósito último foi alcançado, oferecendo evidências de adequação psicométrica acerca de uma medida que poderá ser adequadamente empregada no contexto brasileiro para conhecer os correlatos do bullying.

Apesar do anteriormente comentado, parece pertinente pontuar algumas limitações potenciais destes estudos. A propósito, ressalta-se inicialmente o fato de as amostras utilizadas terem sido de conveniência (não probabilísticas), contando com a colaboração daqueles que concordaram em colaborar com o estudo, o que poderá restringir a generalização dos achados quanto à presença de bullying no contexto de estudo. Além disto, consideraram-se tão somente estudantes de instituições de ensino que permitiram a aplicação, independente da incidência deste fenômeno nos ambientes escolares respectivos. Contudo, alerta-se que o propósito último não foi exatamente estudar o bullying em si, mas adaptar uma medida que permitirá, no futuro, avaliá-lo. Neste sentido, o número e a natureza dos participantes foram suficientes para os propósitos psicométricos delineados (Pasquali, 2003).

Para além do anteriormente comentado, destaca-se ainda como uma limitação potencial o emprego de medidas de autorrelato, isto é, do tipo "lápis e papel”, implicando em algumas desvantagens, visto que o participante pode falsear suas respostas, diferindo-as da realidade (Kohlsdorf \& Costa, 2009) ou tê-las enviesada devido à desejabilidade social, principalmente quando o tema é considerado indesejável socialmente, como é o caso do bullying (Salmivalli, Lagerspetz, Björkqvist, Österman, \& Kaukiainen, 1996). Porém, esta não é uma dificuldade específica da escala tratada, mas uma propriedade das medidas que têm esta natureza, fomentando-se o emprego de estratégias alternativas, como aquelas de medidas implícitas (Gouveia, Athayde, Mendes, \& Freire, 2012).

Quanto aos achados principais, no Estudo 1 foi possível reunir evidências psicométricas da ECVB. Observou-se, por exemplo, que seus itens foram discriminativos, mesmo consideran- 
do o critério mais estrito da mediana (Pasquali, 2003), tendo a estrutura unidimensional teorizada emergido sem qualquer imposição, isto é, a partir da análise de componentes principais, demonstrando que unicamente um fator foi suficiente para explicar a maior parte da variância do construto, como ficou visível por meio do critério robusto da análise paralela (Hayton et al., 2004). Além disso, seu alfa de Cronbach foi superior ao ponto de corte comumente adotado $(0,70$; Pasquali, 2003), que é endossado pela homogeneidade do conjunto de itens, cuja média das correlações inter-itens foi superior ao que se recomenda como aceitável $(0,20$; Clark \& Watson, 1995). Estes achados foram coerentes com aqueles descritos por outros autores (Atik \& Guneri, 2012; Felix et al., 2011), reforçando a qualidade psicométrica desta medida.

No que se referiu ao Estudo 2, a análise fatorial confirmatória impondo um único fator ofereceu indicadores de ajuste satisfatórios, superando os pontos de corte frequentemente recomendados na literatura (e.g., CFI $>0,90$ e RMSEA $<0,08$; Tabachnick \& Fidell, 2013). Portanto, parece inconteste no contexto brasileiro a presença de um fator geral de bullying, como avaliado pela Escala Califórnia de Vitimização do Bullying. Seu alfa de Cronbach apresentou coeficiente superior àqueles de outros estudos, como, por exemplo, o de Atik e Guneri (2012), que se situou em 0,72. Outro indicador de consistência interna, a confiabilidade composta, apresentou coeficiente adequado, situando-se próximo a 0,80 (Fornell \& Larcker, 1981); também a homogeneidade, em linha com o estudo anterior, ofereceu resultados que corroboraram este parâmetro (Clark \& Watson, 1995).

Por fim, ressalta-se a possibilidade de estudos futuros com a temática bullying. Primeiramente, estudos ora descritos poderão ser replicados, diversificando as amostras quanto à idade dos participantes (e.g., incluindo menores de nove anos), conhecer evidências de estabilidade temporal desta medida (teste-reteste), avaliar em que medida pode estar relacionada com indicadores com os quais se esperaria alguma associação, a exemplo de agressão, mas também com outros que podem ter impacto na vida de vítimas e agressores, a exemplo da ansiedade e depressão. Como um fenômeno que retrata um contexto cultural determinado, talvez seja pertinente conhecer em que medida este tipo de conduta pode ser mais recorrente em função dos valores que os jovens assumem como princípios que guiam sua vida. Caberá, ainda, conhecer a incidência deste tipo de conduta nas escolas, favorecendo seu acompanhamento e permitindo avaliar estratégias de intervenção que visem a reduzi-lo. Por fim, haverá que pensar em estratégias menos diretas e inibidoras de mensurar este fenômeno, quiçá recorrendo às medidas implícitas (Gouveia et al., 2012).

\section{Referências}

Atik, G. (2011). Assessment of school bullying in Turkey: A critical review of self-report instruments. Procedia Social and Behavioral Sciences, 15, 3232-3238. doi:10.1016/j.sbspro.2011.04.277

Atik, G., \& Guneri, O. Y. (2012). California Bullying Victimization Scale: Validity and reliability evidence for the Turkish middle school children. Procedia - Social and Behavioral Sciences, 46, 1237-1241. doi:10.1016/j.sbspro.2012.05.281

Barbosa, G. A., Gouveia, V. V., \& Barbosa, A. G. (2003). Escalas de avaliação em psiquiatria da infância e da adolescência. In F. B. Assumpção Júnior \& E. Kuczynski (Eds.), Tratado de psiquiatria da infância e da adolescência (pp. 121137). São Paulo, SP: Atheneu.

Biddle, S., Markland, D., Gilbourne, D., Chatzisarantis, N., \& Sparkes, A. (2001). Research methods in sport and exercise psychology: Quantitative and qualitative issues. Journal of Sports Sciences, 19, 777-809. doi:10.1080/026404101317015438

Clark, L. A., \& Watson, D. (1995). Constructing validity: Basic issues in objective scale development. Psychological Assessment, 7, 309-319. doi:10.1037/1040-3590.7.3.309

Cornell, D. G., Sheras, P. L., \& Cole, J. C. (2006). Assessment of bullying. In $\mathrm{S}$ Jimerson \& $\mathrm{M}$. Furlong (Eds.), Handbook of school violence and school safety (pp. 191-210). Mahwah, NJ: Erlbaum.

Diamantopoulos, A., \& Siguaw, J. A. (2000). Introducing LISREL. London: Sage. 
Felix, E. D., Sharkey, J. D., Green, J. G., Furlong, M. J., \& Tanigawa, D. (2011). Getting precise and pragmatic about the assessment of bullying: The development of the California Bullying Victimization Scale. Aggressive Behavior, 37, 234-247. doi:10.1002/ab.20389

Fornell, C., \& Larcker, D. F. (1981). Evaluating structural equation models with unobservable variables and measurement error. Journal of Marketing Research, 18, 39-50. doi:10.2307/3151312

Gouveia, V. V., Athayde, R. A. A., Mendes, L. A., \& Freire, S. E. A. (2012). Introdução às medidas implícitas: Conceitos, técnicas e contribuições. Diaphora, 12, 3-16.

Hair, J. F., Black, W. C., Babin, B. J., Anderson, R. E., \& Tatham, R. L. (2009). Análise multivariada de dados. Porto Alegre, RS: ArtMed

Hayton, J. C., Allen, D. G., \& Scarpello, V. (2004). Factor retention decisions in exploratory factor analysis: A tutorial on parallel analysis. Organizational Research Methods, 7, 191-205. doi:10.1177/1094428104263675

Hooper, D., Coughlan, J., \& Mullen, M. R. (2008). Structural equation modelling: Guidelines for determining model fit. The Electronic Journal of Business Research Methods, 6, 53-60.

Hoover, J. H., Oliver, R., \& Hazler, R. J. (1992). Bullying: Perceptions of adolescent victims in the Midwestern USA. School Psychology International, 13, 5-16. doi:10.1177/0143034392131001

Kohlsdorf, M., \& Costa, A. L., Jr. (2009). O autorrelato na pesquisa em Psicologia da Saúde: Desafios metodológicos. Psicologia Argumento, 27, 131-139.

Lee, T., \& Cornell, D. (2010). Concurrent validity of the Olweus Bully/Victim Questionnaire. Journal of School Violence, 9, 1-18. doi:10.1080/15388220903185613

Leymann,H.(1990). Mobbing and psychological terror at workplaces. Violence and Victims, 5, 119-126.

Lisboa, C., Braga, L., \& Ebert, G. (2009). O fenômeno bullying ou vitimização entre pares na atualidade: Definições, formas de manifestação e possibilidades de intervenção. Contextos Clínicos, 2, 59-71. doi:10.4013/ctc.2009.21.07

Olweus, D. (1978). Aggression in the schools: Bullies and whipping boys. New York: Wiley.

Olweus, D. (1984). Aggressors and their victims: Bullying at school. In N. Frude \& H. Gault
(Eds.), Disruptive behaviour in schools (pp. 5776). New York: Wiley and Sons.

Olweus, D. (1996). The Revised Olweus Bully/ Victim Questionnaire. Mimeo. Bergen, Norway: Research Center for Health Promotion, University of Bergen.

Pasquali, L. (2003). Psicometria: Teoria dos testes na Psicologia e na Educação. Petrópolis, RJ: Vozes.

Rigby, K. (2008). Children and bullying: How parents and educators can reduce bullying at school. Boston, MA: Blackwell.

Sairanen, L., \& Pfeffer, K. (2011). Self-reported handling of bullying among junior high school teachers in Finland. School Psychology International, 32, 330-344. doi:10.1177/0143034311401795

Salmivalli, C. (2010). Bullying and the peer group: A review. Aggression and Violent Behavior, 15, 112-120. doi:10.1016/j.avb.2009.08.007

Salmivalli, C., Lagerspetz, K., Björkqvist, K., Österman, K., \& Kaukiainen, A. (1996). Bullying as a group process: Participant roles and their relations to social status within the group. Aggressive Behavior, 22, 1-15. doi:10.1002/(SICI)10982337(1996)22:1<1::AID-AB1>3.0.CO;2-T

Sansone, R. A., \& Sansone, L. A. (2008). Bully victims: Psychological and somatic aftermaths. Psychiatry, 5, 62-64.

Siyez, D. M., \& Kaya, A. (2008). Validity and reliability of the Brief Multidimensional Student's Life Satisfaction Scale with Turkish children. Journal of Psychoeducational Assessment, 26, 139-147. doi:10.1177/0734282907307802

Tabachnick, B. G., \& Fidell, L. S. (2013). Using multivariate statistics $\left(6^{\text {th }}\right.$ ed.). Boston, MA: Allyn and Bacon.

Vanderbilt, D., \& Augustyn, M. (2010). The effects of bullying. Paediatrics and Child Health, 20, 315-320

Zapf, D. (1999). Organizational, work group related and personal causes of mobbing/bullying at work. International Journal of Manpower, 20, 70-85. doi:10.1108/01437729910268669

Recebido: 21/10/2013

$1^{a}$ revisão: $22 / 05 / 2014$

$2^{a}$ revisão: $26 / 09 / 2014$

Aceite final: 29/09/2014 